Portland State University

PDXScholar

Physics Faculty Publications and Presentations

Physics

8-8-2018

\title{
Supercanonical Probability Distributions
}

John D. Ramshaw

Portland State University, jdramshaw@yahoo.com

Follow this and additional works at: https://pdxscholar.library.pdx.edu/phy_fac

Part of the Physics Commons

Let us know how access to this document benefits you.

\section{Citation Details}

Ramshaw, J. D. (2018). Supercanonical probability distributions. Physical Review E, 98(2), 020103.

This Article is brought to you for free and open access. It has been accepted for inclusion in Physics Faculty Publications and Presentations by an authorized administrator of PDXScholar. Please contact us if we can make this document more accessible: pdxscholar@pdx.edu. 


\title{
Supercanonical probability distributions
}

\author{
John D. Ramshaw \\ Department of Physics, Portland State University, Portland, Oregon 97207, USA
}

(Received 19 February 2018; published 8 August 2018)

\begin{abstract}
The canonical probability distribution describes a system in thermal equilibrium with an infinite heat bath. When the bath is finite the distribution is modified. These modifications can be derived by truncating a Taylor-series expansion of the entropy of the heat bath, but their form depends on the expansion parameter chosen. We consider two such expansions, which yield supercanonical (i.e., higher-order canonical) distributions of exponential and power-law form. The latter is identical in form to the "Tsallis distribution," which is therefore a valid asymptotic approximation for an arbitrary finite heat bath, but bears no intrinsic relation to Tsallis entropy.
\end{abstract}

DOI: 10.1103/PhysRevE.98.020103

The probability that a system in thermal equilibrium with an infinite heat bath occupies microstate $i$ is given by the canonical distribution

$$
p_{i}=Z^{-1} \exp \left\{-\beta E_{i}\right\}
$$

where $E_{i}$ is the energy of microstate $i, \beta=1 / T_{b}, T_{b}$ is the temperature of the heat bath in energy units, and $Z(\beta) \equiv \sum_{i}$ $\exp \left\{-\beta E_{i}\right\}$ is the canonical partition function. Equation (1) is widely regarded as the single most important formula in statistical physics. Our purpose here is to explore the corrections or modifications to it that arise when the heat bath is finite. Such corrections represent an important aspect of the thermodynamics and statistical mechanics of small systems [1-3], which are experiencing a lively renaissance (e.g., [4-8]) stimulated in part by rapid advances in nanotechnology. Much of the literature on finite heat baths has been narrowly focused on the observation that simple ideal heat baths imply power-law probability distributions of the same form as those obtained by extremizing the Tsallis entropy [9-11]. This correspondence has often been interpreted as evidence in support of the Tsallis entropy, but that inference is unjustified [12].

The probability distribution for a system in thermal equilibrium with a finite heat bath is fundamentally given by [13-15]

$$
p_{i}=\frac{W_{b}\left(E_{0}-E_{i}\right)}{\sum_{j} W_{b}\left(E_{0}-E_{j}\right)},
$$

where $E_{0}$ is the total energy of the system and bath together, and $W_{b}(E)$ is the number of bath states with energies in the interval $(E, E+\Delta E)$, where $\Delta E$ is a macroscopically small but finite energy tolerance. Equation (2) is based on the hypothesis of equal a priori probabilities, according to which those bath states are presumed to be equally probable, and the further presumption that the heat bath is sufficiently large and complex that $W_{b}(E) \cong \omega_{b}(E) \Delta E$, where $\omega_{b}(E)$ is a differentiable continuous approximation to the true discontinuous density of bath states [15]. If the heat bath were isolated with energy $E$, its entropy $S_{b}$, temperature $T_{b}$, and heat capacity $C_{b}$ would be given by

$$
\begin{gathered}
S_{b}(E)=\log W_{b}(E), \\
\frac{1}{T_{b}(E)}=\frac{\partial S_{b}}{\partial E},
\end{gathered}
$$

and $C_{b}(E) \equiv\left(\partial T_{b} / \partial E\right)^{-1}$. Note that entropy is dimensionless when temperature is expressed in energy units, which is formally equivalent to replacing Boltzmann's constant by unity. If the heat bath is a proper thermodynamic system, then as it becomes infinitely large $T_{b}(E)$ will approach a finite limiting value, while $E$ itself and $C_{b}(E)$ become infinite. The latter divergence reflects the fact that it requires infinite energy to produce a finite change in the temperature of an infinite system.

The standard derivation of the canonical distribution from Eq. (2) is based on the assumption that the heat bath is much larger than the system, so that $E_{i} \ll E_{0}$ and a Taylor series expansion of $S_{b}\left(E_{0}-E_{i}\right)=\log W_{b}\left(E_{0}-E_{i}\right)$ in powers of $E_{i}$ can reasonably be presumed to converge rapidly [13]. Equation (1) is then an immediate consequence of truncating this expansion at the linear term [13-15]. However, this procedure further implies that Eq. (1) is not exact but is in principle modified by the higher-order terms in the expansion, at least for finite values of $E_{0}$. It is instructive and enlightening to examine the form of those modifications when the quadratic term of order $E_{i}^{2}$ [13-16] is retained. Thus we proceed to explore the implications of the approximation

$$
S_{b}\left(E_{0}-E_{i}\right)=S_{b}\left(E_{0}\right)-\beta_{1} E_{i}-\beta_{2} E_{i}^{2},
$$

where

$$
\begin{gathered}
\beta_{1} \equiv\left(\frac{\partial S_{b}}{\partial E}\right)_{0}=\frac{1}{T_{b}^{0}}, \\
\beta_{2} \equiv-\frac{1}{2}\left(\frac{\partial^{2} S_{b}}{\partial E^{2}}\right)_{0}=\frac{\beta_{1}}{2 C_{b}^{0} T_{b}^{0}},
\end{gathered}
$$

subscript zero denotes evaluation at $E=E_{0}, T_{b}^{0} \equiv T_{b}\left(E_{0}\right)$, and $C_{b}^{0} \equiv C_{b}\left(E_{0}\right)$. Combining Eqs. (2), (3), and (5), we obtain

$$
p_{i}=Z_{2}^{-1} \exp \left\{-\beta_{1} E_{i}-\beta_{2} E_{i}^{2}\right\},
$$

where

$$
Z_{2}\left(\beta_{1}, \beta_{2}\right) \equiv \sum_{i} \exp \left\{-\beta_{1} E_{i}-\beta_{2} E_{i}^{2}\right\} .
$$

It follows from Eq. (7) that $\beta_{2}$ vanishes for an infinite heat bath, whereupon $Z_{2}\left(\beta_{1}, \beta_{2}\right)$ reduces to $Z(\beta)$ and Eq. (8) reduces to Eq. (1). If higher-order terms had been retained in Eq. (5), 
they too would clearly vanish in the same limit. The canonical distribution therefore becomes exact when the heat bath is infinite, but not otherwise. Its universality derives from the fact that the only bath parameter it depends upon is the temperature.

According to Eq. (8), the probabilities $p_{i}$ are unequal, so the entropy $S$ of the system must be evaluated as the BoltzmannGibbs-Shannon (BGS) entropy

$$
S=-\sum_{i} p_{i} \log p_{i},
$$

which contrary to a common misconception is not uniquely or inherently associated with a canonical (or any other) probability distribution [15]. Combining Eqs. (8) and (10), we obtain

$$
S=\beta_{1} \bar{E}+\beta_{2} \overline{E^{2}}+\log Z_{2},
$$

where $\bar{Q} \equiv\left\langle Q_{i}\right\rangle \equiv \sum_{i} p_{i} Q_{i}$. When $\beta_{2}=0$, Eq. (11) reduces to the standard canonical expression for the entropy $S$. The quadratic term in Eq. (5) therefore introduces an additional term $\beta_{2} \overline{E^{2}}$ into the entropy of the system. It follows from Eq. (9) that

$$
\begin{gathered}
\frac{\partial \log Z_{2}}{\partial \beta_{1}}=-\bar{E}, \\
\frac{\partial \log Z_{2}}{\partial \beta_{2}}=-\overline{E^{2}},
\end{gathered}
$$

which combine with Eq. (11) to imply that

$$
d S=\beta_{1} d \bar{E}+\beta_{2} d \overline{E^{2}} .
$$

Note, however, that $\overline{E^{2}}$ is not an independent variable, because $\bar{E}$ and $\overline{E^{2}}$ are both functions of $E_{0}$, so $d \bar{E}$ and $d \overline{E^{2}}$ are both simply proportional to $d E_{0}$ and therefore to each other. Thus $d S$ is simply proportional to $d \bar{E}$, so that the second law of thermodynamics remains valid in the form $d \bar{E}=T d S$, which defines and determines the temperature $T$ of the system. Thus

$$
\frac{1}{T}=\frac{d S}{d \bar{E}}=\beta_{1}+R \beta_{2}=\frac{1}{T_{b}^{0}}\left(1+\frac{R}{2 C_{b}^{0} T_{b}^{0}}\right),
$$

where

$$
R \equiv \frac{d \overline{E^{2}}}{d \bar{E}}=\frac{d \overline{E^{2}} / d E_{0}}{d \bar{E} / d E_{0}}
$$

The parameters $\beta_{1}$ and $\beta_{2}$ are functions of $E_{0}$ via Eqs. (6) and (7), so $T$ can be evaluated by substituting Eq. (9) into Eqs. (12) and (13), differentiating the results with respect to $E_{0}$ using the chain rule, substituting the resulting expressions for $d \bar{E} / d E_{0}$ and $d \overline{E^{2}} / d E_{0}$ into Eq. (16) to obtain $R$, and combining the result with Eq. (15). Unfortunately, the resulting expression for $T$ is so complicated that it does not seem useful or enlightening. Note, however, that Eq. (15) implies that $T \neq T_{b}^{0}$ unless the heat bath is infinite, in which case $\beta_{2} \rightarrow 0$ and $C_{b}^{0} \rightarrow \infty$ as discussed above.

The truncated series expansion in Eq. (5) could readily be extended to higher order if desired, but the resulting formalism rapidly becomes so cumbersome that it seems unlikely to be useful. It is more fruitful to observe that $E_{i}$ is not the only possible variable in powers of which $S_{b}\left(E_{0}-E_{i}\right)$ might usefully be approximated by a truncated Taylor series. As is well known,
$W_{b}(E)$ in finite ideal systems, such as ideal gases or harmonic solids, is typically proportional to $E^{\alpha}$, where the exponent $\alpha$ is on the order of the number of particles or degrees of freedom of the system [9,13-15]. This suggests that faster convergence might be obtained by regarding $S_{b}(E)=\log W_{b}(E)$ as a function of $\log E$ rather than $E$ itself. We therefore proceed to approximate $S_{b}(E)-S_{b}\left(E_{0}\right)$ by a truncated Taylor series expansion in powers of $\log E-\log E_{0}$. Since $E_{0}$ will remain large but finite, the lowest-order correction to the canonical distribution is obtained by truncating the expansion at the linear term. Thus we write

$$
\begin{aligned}
S_{b}(E) & =S_{b}\left(E_{0}\right)+\alpha\left(\log E-\log E_{0}\right) \\
& =S_{b}\left(E_{0}\right)+\log \left(\frac{E}{E_{0}}\right)^{\alpha}
\end{aligned}
$$

where

$$
\alpha \equiv\left(\frac{\partial S_{b}}{\partial \log E}\right)_{0}=E_{0}\left(\frac{\partial S_{b}}{\partial E}\right)_{0}=E_{0} \beta_{1}=\frac{E_{0}}{T_{b}^{0}}
$$

and use has been made of Eq. (6). Combining Eqs. (2), (3), (17), and (18), we obtain

$$
p_{i}=\widehat{Z}^{-1}\left(1-\frac{\beta_{1} E_{i}}{\alpha}\right)^{\alpha},
$$

where

$$
\widehat{Z}\left(\alpha, \beta_{1}\right) \equiv \sum_{j}\left(1-\frac{\beta_{1} E_{j}}{\alpha}\right)^{\alpha} .
$$

Aside from minor notational differences, Eq. (19) is of precisely the same form as the so-called Tsallis distribution previously obtained by extremizing the Tsallis entropy [17], and subsequently legitimized by the observation that it describes a system in equilibrium with an ideal finite heat bath for which $W_{b}(E)$ has the same power-law form [9]. The present derivation shows that this distribution is actually much more general: it is not restricted to ideal heat baths but rather represents a first-order asymptotic approximation to $S_{b}(E)$ for an arbitrary finite heat bath with an arbitrary quasicontinuous density of states. This derivation also provides exact expressions for the parameters $\alpha$ and $\beta_{1}$ in terms of the caloric equation of state $T_{b}(E)$ of the heat bath and the total energy $E_{0}$. Moreover, the systematic expansion procedure on which this approximation is based could in principle be extended to higher order to generate a sequence of increasingly accurate controlled approximations.

The general validity of Eq. (19) as an asymptotic approximation to $p_{i}$ for an arbitrary finite heat bath, together with the associated exact expressions for the parameters $\alpha$ and $\beta_{1}$ therein, constitute the principal result of this Rapid Communication. This result implies that the power-law probability distribution of Eq. (19) is no less general, and indeed is quite likely more accurate for finite $E_{0}$, than the usual canonical distribution of Eq. (1), to which Eq. (19) clearly reduces in the limit $\alpha \rightarrow \infty$ or $E_{0} \rightarrow \infty$. Thus, in spite of their quite different mathematical character, Eqs. (1) and (19) are birds of a feather; they are both based on truncating a Taylor series expansion of $S_{b}(E)$ about the point $E=E_{0}$ at the linear term, but with respect to different variables. 
Unfortunately, the thermodynamic relations implied by the power-law distribution of Eq. (19) are more complicated than those for the exponential distribution of Eq. (8). Combining Eqs. (10) and (19), we obtain

$$
S=-\alpha\left\langle\log \left(1-\frac{\beta_{1} E_{i}}{\alpha}\right)\right\rangle+\log \widehat{Z} .
$$

It follows from Eqs. (19) and (20) that

$$
\begin{gathered}
\frac{\partial \log \widehat{Z}}{\partial \alpha}=\left\langle\log \left(1-\frac{\beta_{1} E_{i}}{\alpha}\right)\right\rangle+\frac{\beta_{1}}{\alpha}\left\langle\frac{E_{i}}{1-\beta_{1} E_{i} / \alpha}\right\rangle, \\
\frac{\partial \log \widehat{Z}}{\partial \beta_{1}}=-\left\langle\frac{E_{i}}{1-\beta_{1} E_{i} / \alpha}\right\rangle .
\end{gathered}
$$

Combining Eqs. (21)-(23), we obtain

$$
S=-\alpha \frac{\partial \log \widehat{Z}}{\partial \alpha}-\beta_{1} \frac{\partial \log \widehat{Z}}{\partial \beta_{1}}+\log \widehat{Z} .
$$

As before, the temperature $T$ of the system could in principle be evaluated as $T=(d S / d \bar{E})^{-1}$, where $S$ is given by Eq. (24), but the result is again so complicated that it seems of dubious utility or interest. Of course, there is no need to evaluate $T$ in order to make use of the probability distribution of Eq. (19); it must simply be remembered that according to Eq. (6) the parameter $\beta_{1}$ is fundamentally related to the temperature $T_{b}^{0}=T_{b}\left(E_{0}\right)$ of the heat bath evaluated at the total energy $E_{0}$, and not to the temperature $T$ of the system itself.

As is well known, the canonical distribution of Eq. (1) satisfies and can be derived from a principle of maximum entropy $[15,18,19]$, so the question naturally arises as to whether the supercanonical distributions of Eqs. (8) and (19) can likewise be derived from such a principle. The answer is a qualified "yes," but it must be kept in mind that the probability distribution $p_{i}$ is fundamentally determined by Eq. (2), the validity of which is neither derived from nor contingent on a maximum entropy principle. On the contrary, maximum entropy principles derive their validity from the probability distribution, not vice versa [20], and they obviously depend critically on both the form of the entropy and the constraints imposed upon its maximization. The present development is based on the BGS entropy $S$ of Eq. (10), which we regard as sacrosanct, but the probability distribution obtained by maximizing it still depends upon the constraints. When the only constraint (apart from normalization) is on the value of the mean energy $\bar{E}=\left\langle E_{i}\right\rangle$, the canonical distribution of Eq. (1) results. It is easy to verify that the exponential supercanonical distribution of Eq. (8) results when an additional constraint is imposed on the mean-square energy $\overline{E^{2}}=\left\langle E_{i}^{2}\right\rangle$. This is simply the second-order special case of the maximum entropy method of moments [21]. It is also easy to verify that the power-law supercanonical distribution of Eq. (19) results when $S$ is maximized subject to a constraint on the value of $\left\langle\log \left(E_{0}-E_{i}\right)\right\rangle$, but the physical significance of that unusual constraint is not immediately obvious (cf. [22]). Of course, it is well known that Eq. (19) can also be obtained by maximizing the Tsallis entropy subject to a conventional constraint on $\bar{E}$ [17]. However, the fundamental significance of that result seems dubious, because it is straightforward to determine a function $\sigma(p)$ such that the extremization of $\sum_{i} \sigma\left(p_{i}\right)$ subject to a constraint on $\bar{E}$ will reproduce an arbitrary probability distribution of the form $p_{i}=p\left(E_{i}\right)$. Any monotonic function of $\sum_{i} \sigma\left(p_{i}\right)$ then defines an "entropy" with the same extremum property. It is straightforward to confirm that the Tsallis and Rényi entropies can be constructed from Eq. (19) in precisely this way, but this is a mere mathematical exercise with no physical content.

Finally, we emphasize that the deviations from the canonical distribution that occur when the heat bath is finite do not in any sense invalidate or necessitate modifications to or "generalizations" of the BGS entropy of Eq. (10), as has often been suggested by the advocates of Tsallis entropy. The terminology "Tsallis distribution" therefore seems unfortunate and inappropriate, since the power-law distribution of Eq. (19) does not depend in any way on the Tsallis entropy for its general validity or significance.
[1] T. L. Hill, Thermodynamics of Small Systems (Dover, New York, 1994).

[2] J. S. Rowlinson, J. Chem. Soc. Faraday Trans. 2 82, 1801 (1986).

[3] T. L. Hill, Nano Lett. 1, 273 (2001).

[4] Nonequilibrium Statistical Physics of Small Systems: Fluctuation Relations and Beyond, edited by R. Klages, W. Just, and C. Jarzynski (Wiley-VCH, Weinheim, 2013).

[5] P. D. Dixit, J. Chem. Phys. 138, 184111 (2013).

[6] M. Khanpour, L. A. Rivera-Rivera, and T. D. Sewell, Phys. Chem. Liq. 53, 467 (2015).

[7] E. N. Miranda, Eur. J. Phys. 38, 065101 (2017).

[8] R. Uzdin, Phys. Rev. E 96, 032128 (2017).

[9] A. R. Plastino and A. Plastino, Phys. Lett. A 193, 140 (1994).

[10] M. P. Almeida, Physica A 300, 424 (2001).

[11] F. Q. Potiguar and U. M. S. Costa, Physica A 344, 614 (2004), and references cited therein.

[12] J. D. Ramshaw, Phys. Lett. A 198, 122 (1995).
[13] R. Becker, Theory of Heat, 2nd ed. (Springer-Verlag, New York, 1967).

[14] R. Kubo, Statistical Mechanics: An Advanced Course with Problems and Solutions (North-Holland, Amsterdam, 1971).

[15] J. D. Ramshaw, The Statistical Foundations of Entropy (World Scientific, Singapore, 2018).

[16] A. I. Khinchin, Mathematical Foundations of Statistical Mechanics (Dover, New York, 1949).

[17] C. Tsallis, J. Stat. Phys. 52, 479 (1988).

[18] The Maximum Entropy Formalism, edited by R. D. Levine and M. Tribus (MIT Press, Cambridge, MA, 1979).

[19] E. T. Jaynes: Papers on Probability, Statistics and Statistical Physics, edited by R. D. Rosenkrantz (Reidel, Dordrecht, 1983).

[20] J. D. Ramshaw, Eur. J. Phys. 39, 035104 (2018).

[21] L. R. Mead and N. Papanicolaou, J. Math. Phys. 25, 2404 (1984).

[22] E. W. Montroll and M. F. Shlesinger, J. Stat. Phys. 32, 209 (1983). 